Arch. Math. 86 (2006) 26-30

0003-889X/06/010026-05

DOI 10.1007/s00013-005-1481-0

(C) Birkhäuser Verlag, Basel, 2006

Archiv der Mathematik

\title{
Free actions of virtually FC-groups
}

\author{
By
}

MARTIN R. PETTET

\begin{abstract}
If an infinite group $G$ admits a free action by a group of automorphisms $A$ which is virtually an FC-group and which has only finitely many orbits, then $G$ is isomorphic to the additive group of a field and the action is that of a group of semilinear transformations.
\end{abstract}

The $F C$-centre $\Delta(A)$ of a group $A$ is the subgroup consisting of all elements $\alpha \in A$ for which the conjugacy class $\alpha^{A}$ of $\alpha$ in $A$ is finite (i.e., those elements $\alpha$ for which the centralizer $C_{A}(\alpha)$ has finite index in $\left.A\right)$. $A$ is said to be an $F C$-group if $A=\Delta(A)$. If $A$ acts as a group of automorphisms on a group $G$, it is said to act freely if the stabilizer $C_{A}(g)$ of every element $g \in G^{\#}=G \backslash\{1\}$ is trivial. The main purpose of this note is to record a short, almost self-contained proof of the following:

Theorem. Let $G$ be an infinite group admitting a group of automorphisms A that acts freely on $G$ and has $r$ orbits in $G^{\#}=G \backslash\{1\}, r<\infty$. If A contains an $F C$-subgroup of finite index, there is a (commutative) field $E$ such that

(a) $G$ is isomorphic to the additive group of $E$,

(b) $\Delta(A)$ is isomorphic to a subgroup of index $r|A: \Delta(A)|$ in the multiplicative group $E^{\times}=E \backslash\{0\}$,

(c) $A / \Delta(A)$ is isomorphic to a group $\mathcal{A}$ of (field) automorphisms of $E$,

(d) under the identification of $E^{+}$with $G$, A corresponds to a group of semilinear transformations $x \mapsto a x^{\sigma}, x \in E, a \in E^{\times}, \sigma \in \mathcal{A}$.

The theorem may be viewed as a generalization of a theorem of W. Kreft [3] on nearfields, the result there corresponding to the case that $A$ is an FC-group whose action on $G^{\#}$ is transitive (Corollary 1). However, the argument presented here is entirely group theoretic.

Proof of the the ore m. Because $\Delta(A)$ contains every FC-subgroup of finite index in $A,|A: \Delta(A)|<\infty$. Note that if $B$ is any subgroup of finite index in $A$ and $T$ is a left 
transversal for $B$ in $A$ (so $A=\bigcup_{\alpha \in T} \alpha B$ ), then each non-trivial $A$-orbit $g^{A}$ is a disjoint union of the $B$-orbits $\left(g^{\alpha}\right)^{B}, \alpha \in T$, and so the number of $B$-orbits in $G^{\#}$ is $r|A: B|<\infty$. We begin with two observations about the action of $A$, the first of which is a slight modification of an argument in [4].

(i) If $|A: B|<\infty$ and $H$ is a $B$-invariant subgroup of $G$ then, for any non-identity element $\alpha$ of $B \cap \Delta(A)$, the map $h \mapsto h^{-1} h^{\alpha}$ for $h \in H$ is a bijection from $H$ to itself.

Because $A$ acts freely, the map $\theta_{\alpha}: G \rightarrow G$ defined by $\theta_{\alpha}: g \mapsto g^{-1} g^{\alpha}$ is certainly injective. If $C=C_{B}(\alpha)$, then $|A: C| \leqq|A: B|\left|A: C_{A}(\alpha)\right|<\infty$ and so there are only finitely many $C$-orbits in $G$. Since $\theta_{\alpha}$ maps $C$-orbits to $C$-orbits, its restriction to $H$ is a bijection from $H$ to itself, as claimed.

(ii) If $B \leqq A$ with $|A: B|<\infty$, then $G$ contains no proper, non-trivial $B$-invariant subgroups.

$|A: B \cap \Delta(A)| \leqq|A: B||A: \Delta(A)|<\infty$ and so we may assume that $B=B \cap \Delta(A) \leqq$ $\Delta(A)$. Let $H$ be a non-trivial $B$-invariant subgroup of $G$. Each coset of $H$ is infinite but the number of $B$-orbits is finite and so if $x \in G$ then $x \neq x^{\beta} \in x H$ for some $\beta \in B^{\#}$. Then $x^{-1} x^{\beta} \in H$, whence, by (i), $x^{-1} x^{\beta}=h^{-1} h^{\beta}$ for some $h \in H$. Therefore, $\left(x h^{-1}\right)^{\beta}=x h^{-1}$ and, since $A$ acts freely, $x=h \in H$. We conclude that $H=G$, proving (ii).

Next, we show that $G$ is abelian, again following [4]. Observe that each conjugacy class in $G$ contains at most one element from each $\Delta(A)$-orbit. For if $g, x \in G$ such that $x^{-1} g x=g^{\alpha} \neq g$ for some $\alpha \in \Delta(A)$ then by (i), $x=y^{-1} y^{\alpha}$ for some $y \in G$ and so $\left(y g y^{-1}\right)^{\alpha}=y g y^{-1}$, forcing $g=1$. Hence, the cardinality of each class is bounded above by the number of $\Delta(A)$-orbits. By a theorem of B. H. Neumann (e.g., [5, Theorem 4.35], $G^{\prime}$ is finite and so, because all non-trivial $A$-orbits are infinite, $G^{\prime}=1$.

For the remainder of the argument, we write $G$ additively.

If $X$ is a subset of $\operatorname{End}(G)$ and $\theta \in \operatorname{End}(G)$, let $C_{X}(\theta)$ be the set of all $\eta \in X$ such that $\eta \theta=\theta \eta$. If $E=\left\{\theta \in \operatorname{End}(G):\left|A: C_{A}(\theta)\right|<\infty\right\}$ then $E$ is a subring of $\operatorname{End}(G)$ containing $\Delta(A)$ and is invariant under conjugation by $A$. If $\theta \in E$ and $C=C_{A}(\theta)$, $|A: C|<\infty$ and the kernel and image of $\theta$ are each $C$-invariant subgroups of $G$. By (ii), if $\theta \neq 0$ then $\theta \in \operatorname{Aut}(G)$ and so $E$ is a division ring. Moreover, the multiplicative group $E^{\times}=E \backslash\{0\}$ acts freely on $G$, for if $\theta \in E^{\times}$and $\theta \neq 1$ then $\theta-1 \in E^{\times}$and so $\theta-1$ has trivial kernel.

Fix an element $g \in G^{\#}$. If $\theta \in E^{\times}$, let $K=g^{C_{E}(\theta)}=\left\{g^{\eta}: \eta \in C_{E}(\theta)\right\}$. Because $C_{E}(\theta)$ is an additive group, $K$ is a subgroup of $G$. Moreover, $K$ is invariant under $C_{E^{\times}}(\theta)$ and hence, under $C_{\Delta(A)}(\theta)$. By (ii), $K=G$ and so $C_{E^{\times}}(\theta)$ is transitive on $G^{\#}$. Because $E^{\times}$ acts freely on $G$, it follows that $C_{E^{\times}}(\theta)=E^{\times}$. Therefore, $E^{\times}$is abelian and $E$ is a field.

$G$ may be identified as the additive group $E^{+}$of the field $E$ via the bijection $\theta \mapsto g^{\theta} \in G$ for $\theta \in E$. Because $E^{\times}$acts freely and transitively on $G^{\#},\left|E^{\times}: \Delta(A)\right|=r|A: \Delta(A)|$, the number of orbits of $\Delta(A)$ in $G^{\#}$. From the fact that $\Delta(A) \leqq E^{\times}$and $|A: \Delta(A)|<\infty$, it follows that $C_{A}\left(E^{\times}\right) \leqq C_{A}(\Delta(A))=\Delta(A)$ and so the conjugation action of $A$ induces a faithful action of $A / \Delta(A)$ on $E$ as a group $\mathcal{A}$ of automorphisms. This completes the proof of statements (a), (b) and (c).

Finally, we may define an injective derivation $\sigma: A \rightarrow E^{\times}$(whose restriction to $\Delta(A)$ is the identity map) by $g^{\sigma(\alpha)}=g^{\alpha}$ for all $\alpha \in A$. Then for any $\theta \in E$ and $\alpha \in A$, 
$\left(g^{\theta}\right)^{\alpha}=\left(g^{\alpha}\right)^{\theta^{\alpha}}=g^{\sigma(\alpha) \theta^{\alpha}}$ (where $\left.\theta^{\alpha}=\alpha^{-1} \theta \alpha\right)$ and so the action of $\alpha$ on $E$ induced by the isomorphism between $G$ and $E^{+}$sends $\theta$ to $\sigma(\alpha) \theta^{\alpha}$. Therefore, $A$ is isomorphic to a group of semilinear transformations of $E$, proving (d) and completing the proof of the theorem.

The multiplicative group of a near-field $N$ acts freely and transitively on the non-zero elements of the additive group of $N$. Hence, the case $r=1$ of the theorem may be formulated as a generalization of a result of Kreft [3].

Corollary 1. An infinite near-field whose multiplicative group is a finite extension of an FC-group is a field.

A permutation group $\Gamma$ on a set $\Omega$ is said to be a Frobenius group if it is transitive and all two-point stabilizers are trivial. The rank of $\Gamma$ is the number of orbits in the induced action of $\Gamma$ on $\Omega \times \Omega$ or equivalently, the number of orbits of a point stabilizer $A=\Gamma_{\alpha}$ (where $\alpha \in \Omega$ ) on $\Omega$. The (Frobenius) kernel $G$ consists of the identity together with those elements of $\Gamma$ which have no fixed points. $\Gamma$ is said to be split if this kernel is a (normal) subgroup of $\Gamma$, in which case $A$ is a complement of $G$ in $\Gamma$. If the pair $(G, A)$ satisfies the hypotheses of the theorem, the corresponding semidirect product $\Gamma=A G$ is a split Frobenius group of finite rank on the set $\Omega$ of right cosets of $A$ in $\Gamma$ and so, because neither the additive nor the multiplicative groups of an infinite field can be finitely generated, the theorem may be regarded as an extension of Corollaries 1 and 2 of [4].

Corollary 2. Let $\Gamma$ be an infinite split Frobenius group of finite rank with kernel $G$ and complement $A$. If $A$ is a finite extension of an FC-group then $G$ is abelian, $A$ is abelianby-finite and both groups are infinitely generated.

Although it appears still to be open whether a Frobenius group of arbitrary finite rank with abelian point stabilizers is necessarily split, this is the case if the group has rank two (i.e., if it is sharply doubly transitive). In fact, using near-ring techniques, W. Kerby has shown [2] that a rank two Frobenius group is split if the point stabilizers are FC-groups. Whether this conclusion holds if the stabilizers are only virtually FC will not be resolved here but a purely group theoretic proof of Kerby's result may still be of some interest. The argument below is essentially an elaboration of that used in Theorem 3.4B of [1] to treat the case in which the stabilizers are abelian.

Corollary 3. Let $\Gamma$ be an infinite doubly transitive Frobenius group on the set $\Omega$ and assume that point stabilizers are FC-groups. Then there is a field $E$ such that $\Gamma$ is isomorphic to the affine group Aff $(1, E)$ of maps $E \rightarrow E$ of the form $x \mapsto a x+b,\left(a \in E^{\times}, b \in E\right)$. In particular, $\Gamma$ is split.

Proof. We prove that $\Gamma$ is split, the rest of the corollary being a direct consequence of the theorem.

Let $I=\left\{x \in \Gamma: x^{2}=1 \neq x\right\}$ and let $G$ be the Frobenius kernel. For any $\alpha \in \Omega$, let $Z_{\alpha}=\{1\} \cup\left(I \cap \Gamma_{\alpha}\right)$. The first few observations are well-known.

(i) For any $\alpha, \beta \in \Omega, \alpha \neq \beta$, there exists a unique $t \in I$ such that $\alpha^{t}=\beta$. 
In fact, by hypothesis, there is a unique $t \in \Gamma$ such that $(\alpha, \beta)^{t}=(\beta, \alpha)$. Because $\Gamma_{\alpha} \cap \Gamma_{\beta}=1, t \in I$ and is uniquely determined by $\alpha$ and $\beta$.

(ii) If $\alpha \in \Omega$, the conjugation action of $\Gamma_{\alpha}$ is transitive on $I \backslash \Gamma_{\alpha}$ (and so $I$ is a conjugacy class in $\Gamma$ ).

For if $s, t \in I \backslash \Gamma_{\alpha}$ then, because $\Gamma_{\alpha}$ is sharply transitive on $\Omega \backslash\{\alpha\}, \alpha^{t}=\alpha^{s a}=\alpha^{s^{a}}$ for a unique $a \in \Gamma_{\alpha}$. By (i), $s^{a}=t$.

(iii) $\left|Z_{\alpha}\right| \leqq 2$ (so $Z_{\alpha} \leqq Z\left(\Gamma_{\alpha}\right)$ ).

For suppose $s, t \in I \cap \Gamma_{\alpha}$. If $\beta \neq \alpha$ then by (ii), $s^{b}=t$ for some $b \in \Gamma_{\beta}$ and so $s b=b t$. Thus, $\alpha^{b}=\alpha^{s b}=\alpha^{b t}$, whence $t \in \Gamma_{\alpha} \cap \Gamma_{\alpha^{b}}$. Therefore, $\alpha=\alpha^{b}$ and so $b \in \Gamma_{\alpha} \cap \Gamma_{\beta}=1$ and $s=s^{b}=t$.

(iv) $\Gamma=\Gamma_{\alpha} G$.

It suffices to show that if $\beta \neq \alpha$ then $\Gamma_{\beta} \subseteq \Gamma_{\alpha} G$. By (i), $\beta^{s}=\alpha$ for some $s \in I$ and so if $g \in \Gamma_{\beta}, g^{s} \in \Gamma_{\alpha}$. But $s s^{g} \in G$, for if $s s^{g} \in \Gamma_{\gamma}, \gamma \in \Omega$, then $\gamma^{s}=\gamma^{s^{g}}$ so $s s^{g}=1$ by (i). Therefore, $g=g^{s}\left(s s^{g}\right) \in \Gamma_{\alpha} G$.

(v) If $G \backslash\{1\} \subseteq Z_{\alpha} I$ for some $\alpha \in \Omega$ then $G$ is a subgroup (and so, by (iv), $\Gamma$ is split).

For suppose that $g, h \in G \backslash\{1\}$ such that $g h^{-1} \notin G$. Then a conjugate of $g h^{-1}$ fixes $\alpha$ and so, replacing $g$ and $h$ by corresponding conjugates, we may assume that $g h^{-1} \in \Gamma_{\alpha}$. Write $g=s_{1} t_{1}$ and $h=s_{2} t_{2}$ where $s_{1}, s_{2} \in Z_{\alpha}$ and $t_{1}, t_{2} \in I$. Then $\alpha^{t_{1}}=\alpha^{g}=\alpha^{h}=\alpha^{t_{2}}$ and so by (i), $t_{1}=t_{2}$ and $g h^{-1}=s_{1} s_{2}$. Because $g h^{-1} \neq 1$, (iii) implies that either $s_{1}=1$ and $s_{2} \in I$ or $s_{1} \in I$ and $s_{2}=1$. In the first case, $s_{2}$ is conjugate to $t_{1}=g \in G$ by (ii) and so $s_{2} \in G \cap Z_{\alpha}=1$, a contradiction. In the second, $s_{1}$ is conjugate to $t_{2}=h \in G$, whence $s_{1} \in G \cap Z_{\alpha}=1$, again a contradiction.

By (i), $\Gamma=\Gamma_{\alpha} I$ for any $\alpha \in \Omega$ and so the corollary will follow immediately from the following stronger version of $(\mathrm{v})$ :

(vi) If $G \backslash\{1\} \subseteq \Delta\left(\Gamma_{\alpha}\right) I$ for some $\alpha \in \Omega$ then $\Gamma$ is split.

Suppose that $\Gamma$ is non-split so by (v), $1 \neq g \in G \backslash Z_{\alpha} I$ for some $g \in \Gamma$. Suppose also that $G \backslash\{1\} \subseteq \Delta\left(\Gamma_{\alpha}\right) I$, whence, $1 \neq g t \in \Delta\left(\Gamma_{\alpha}\right)$ for some $t \in I$. For any $\beta \in \Omega \backslash\{\alpha\}$, $\beta^{g t} \neq \beta$ and so (i) implies that $\beta^{g t s_{\beta}}=\beta$ for a unique $s_{\beta} \in I$. Moreover, $s_{\beta} \notin \Gamma_{\alpha}$ (else $s_{\beta} \in Z_{\alpha}$ and $g t s_{\beta} \in \Gamma_{\alpha} \cap \Gamma_{\beta}=1$, whence $g=s_{\beta} t \in Z_{\alpha} I$ ) and so by (ii), $s_{\beta}=a_{\beta} t a_{\beta}^{-1}$ for some $a_{\beta} \in \Gamma_{\alpha}$. Therefore, $a_{\beta}^{(g t)^{-1}} g a_{\beta}^{-1}=g t a_{\beta} t a_{\beta}^{-1}=g t s_{\beta} \in \Gamma_{\beta}$ and hence, $1 \neq\left[a_{\beta},(g t)^{-1}\right] g \in \Gamma_{\beta^{a} \beta}$.

Let $C=C_{\Gamma_{\alpha}}(g t)$ and suppose that $\beta, \gamma \in \Omega$ such that $C a_{\beta}=C a_{\gamma}$ (so $(g t)^{a_{\beta}}=(g t)^{a_{\gamma}}$ ). We claim that $\beta^{C}=\gamma^{C}$. Indeed, $\left[a,(g t)^{-1}\right]=(g t)^{a}(g t)^{-1}$ for any $a \in G$ and so $\left[a_{\beta},(g t)^{-1}\right] g=\left[a_{\gamma},(g t)^{-1}\right] g \in \Gamma_{\beta^{a} \beta} \cap \Gamma_{\gamma^{a_{\gamma}}}$, whence, $\beta^{a_{\beta}}=\gamma^{a_{\gamma}}$. But $\gamma=\beta^{c}$ for some $c \in \Gamma_{\alpha}$ and so $\beta^{a_{\beta}}=\gamma^{a_{\gamma}}=\beta^{c a_{\gamma}}$. Therefore, $a_{\beta} a_{\gamma}^{-1} c^{-1} \in \Gamma_{\alpha} \cap \Gamma_{\beta}=1$ and so $c=a_{\beta} a_{\gamma}^{-1} \in C$, proving the claim.

If $\left|\Gamma_{\alpha}: C\right|=n<\infty$ then because $\Gamma_{\alpha}$ is sharply transitive on $\Omega \backslash\{\alpha\}, C$ has precisely $n$ orbits in $\Omega \backslash\{\alpha\}$. It follows from the preceding paragraph that if $\beta_{i}^{C}, 1 \leqq i \leqq n$ are the $n$ distinct orbits of $C$ in $\Omega \backslash\{\alpha\}$, the cosets $C a_{\beta_{1}}, C a_{\beta_{2}}, \ldots, C a_{\beta_{n}}$ are pairwise distinct and therefore, comprise a complete system of right cosets of $C$ in $\Gamma_{\alpha}$. In particular, $a_{\beta_{i}} \in C$ for some $i$, whence, if $\beta=\beta_{i}, g=\left[a_{\beta},(g t)^{-1}\right] g \in \Gamma_{\beta^{a} \beta}$. This contradicts $g \in G$, completing the proof of (vi) and of the corollary. 
Acknowledgements. The author is indebted to the Department of Mathematics and Statistics at McGill University for its kind hospitality during the preparation of this note.

\section{References}

[1] J. DiXON and B. MoRTIMER, Permutation Groups. 1996.

[2] W. Kerby, On infinite sharply multiply transitive groups. Hamb. Math. Einzelschriften 6, Göttingen 1974.

[3] W. KREFT, Fastkörper mit multiplikativer FC-Gruppe. Abh. Math. Sem. Univ. Hamburg 52, 99-103 (1982).

[4] P. M. NEUMANN and P. J. RowLEY, Free actions of abelian groups on groups. In: Geometry and Cohomology in Group Theory. P. H. Kropholler, G. A. Niblo and R. Stöhr, eds., London Math. Soc. Lecture Note Series 252, 291-295, Cambridge, UK (1998).

[5] D. J. S. RobInson, Finiteness Conditions and Generalized Soluble Groups, Part 1. 1972.

Received: 21 February 2005

Martin R. Pettet

Department of Mathematics

University of Toledo

Toledo, Ohio 43606

USA

mpettet@math.utoledo.edu 